\title{
Key Applications of State-of-the-Art Technologies to Mitigate and Eliminate COVID-19
}

\author{
Sarfraz Nawaz Brohi \\ School of Computer Science and Engineering \\ Taylor's University \\ Selangor, Malaysia \\ sarfraznawaz.brohi@taylors.edu.my
}

\author{
NZ Jhanjhi \\ School of Computer Science and Engineering \\ Taylor's University \\ Selangor, Malaysia \\ noorzaman.jhanjhi@taylors.edu.my
}

\author{
Muhammad Nawaz Brohi \\ Department of Creative Computing \\ Bath Spa University \\ Ras Al Khaimah, UAE \\ mnbrohi@bathspa.ae
}

\author{
Nida Nawaz Brohi \\ Faculty of Medicine and Biomedical Sciences \\ MAHSA University \\ Selangor, Malaysia \\ nida.brohi@outlook.com
}

\begin{abstract}
COVID-19 has stunned the global economy and threatened human life. Due to rapidly emerging fatalities and enormous cases appearing every day, researchers across the globe are producing significant contributions to mitigate this pandemic. Besides the race for discovering a vaccine and treatment for COVID-19, there is utmost focus on flattening the curve by undertaking appropriate measures. The remarkable role of frontline medical practitioners, who are eagerly treating the affected people will be penned in the history books. The efforts of scientists and technologists will be remembered for their extraordinary contributions to assist healthcare professionals and governments in mitigating the threats of COVID-19. Leading technology firms have formed consortiums and research groups, which provide funding and free access to supercomputers for solving complex computational problems to eliminate COVID-19. In this research, we have unveiled five state-of-the-art technologies and their remarkable applications that can be used to mitigate and eliminate the problems of COVID-19. These technologies include Artificial Intelligence (AI), 3D Printing Technology (3DPT), Big Data Analytics (BDA), High Performance Computing (HPC) and Telecommunication Technology (TT). This research investigates the use of technology to encounter COVID-19 and aims to serve as the primary reference for promoting future research as well as developments to produce solutions for COVID-19 using AI, 3DPT, BDA, HPC and TT.
\end{abstract}

Keywords-COVID-19, Pandemic, Artificial Intelligence, 3D Printing Technology, Big Data Analytics, High Performance Computing, Telecommunication Technology

\section{INTRODUCTION}

According to the World Health Organization (WHO), on 31 December 2019, their China office received information about the cases of pneumonia of unknown etiology detected in Wuhan City, Hubei Province of China. WHO was updated by the national authorities in China on 3 January 2020 about the 44 patients infected with the pneumonia of unknown etiology [1]. News about a frightening virus outbreak in China started to appear on various media platforms. On 30 January 2020, WHO declared the outbreak as a Public Health Emergency of international concern [2], and on 11 February 2020, the disease was named as Corona Virus Disease 2019 (COVID-19) [3].
COVID-19 is caused by Severe Acute Respiratory Syndrome Coronavirus 2 (SARS-CoV-2) [4]. The preliminary predictions and claims made by the government officials to sustain economic stability undermined the threats of COVID-19. However, COVID-19 proved to be more than just common flu and, eventually, a Pandemic [5].

COVID-19 is rapidly spreading, and it has conquered the focus of government officials, researchers and scientists. At the time of documenting this research on 11 April 2020, the Johns Hopkins University Coronavirus Resource Centre stated 1,712,674 total confirmed cases and 103,796 deaths from COVID-19 worldwide [6]. Besides being life-threatening, COVID-19 has destabilized businesses, disrupted daily life and created stress as well as anxiety in people. Scientists and researchers have created collaborative platforms to discover a vaccine and medication for COVID-19. However, the rapid creation of vaccination and treatment entails the utmost use of state-of-the-art technologies in the domains such as bioinformatics, epidemiology, genome sequencing and molecular modeling to execute algorithms that require powerful computational capability to address complex queries and discover solutions.

On 2 April 2020, 30 of the world's leading digital technology experts gathered in a virtual roundtable to assist WHO's collaborative response to COVID-19. WHO requested for support from interested technology companies to design and execute ideas and initiatives. WHO received overwhelming support from technology companies to fight the COVID-19 pandemic [7]. In this research, we have unveiled the state-ofthe-art technologies and their key applications that could be used by technologists and scientists to produce exemplary solutions for mitigation and elimination of COVID-19.

\section{Key ApPlicAtions of StATE-OF-THE-ARt TECHNOLOGIES TO Mitigate AND EliminATE COVID-19}

The extensive coverage of literature in the area of COVID19 provided us a wealth of information on several aspects related to science and technology. We further narrowed our research to focus primarily on the role of technology to 
encounter COVID-19. By investigating the impacts of crucial applications of heterogenous technologies, we discovered that Artificial Intelligence (AI), 3D Printing Technology (3DPT), Big Data Analytics (BDA), High Performance Computing (HPC) and Telecommunication Technology (TT) are the five state-of-the-art technologies that could assist the scientists and researchers in mitigating and eliminating COVID-19. The key applications of formerly stated technologies are depicted in Figure 1 and discussed in the following sub-sections.

\section{A. Artificial Intelligence}

AI applications based on deep, machine learning and neural network models are being used as fast-track solutions for prediction and detection of COVID-19. AI systems can detect COVID-19 through chest scans, and they can be used for public mass screening as an alternative to traditional thermometers. These AI-powered systems can be monitored and controlled remotely. AI tools can predict the COVID-19 threats such as detecting the patients that will suffer from lungs damage or severe respiratory problems, which can assist the hospitals in making effective use of their limited resources by prioritizing and attending the patients who need immediate attention. Apart from the health and socioeconomic challenges, the gigantic growth in fake news has been a serious issue during the pandemic. For example, fabricated information regarding government-related policies, restrictions and guidelines, and claims such as turmeric, garlic and lemon can cure COVID-19 have been circulating on social media platforms. Deep learning with graph theory and BDA can be used to detect fake news by analyzing millions of sources. Moreover, the AI-based Apps with geo-location tracking capabilities can be used to track individuals and notify communities via real-time interaction on restrictions, advice and guidelines to avoid COVID-19 hotspots. Most importantly, AI can be used to create drug discovery platforms to identify molecules with potential effects on the coronavirus replication. AI developments such as robots can also be used as support systems to disinfect hospitals, public transport hubs, restaurants and potential COVID-19 hotspots without human interaction with infectious objects. Some noteworthy contributions of AI to encounter COVID-19 are reported in [8 - 10].

\section{B. 3D Printing Technology}

The rapidly expanding daily curve of COVID-19 cases has made it challenging for the countries to meet the equipment demand. There is a shortage of medical devices and Personal Protective Equipment (PPE) for frontline healthcare professionals. During this pandemic, 3DPT has the potential to save lives. Using 3DPT, companies can produce items such as face shields, face masks, ventilators, oxygen masks, test kit swabs and reusable filter masks in a short timeframe. Moreover, the simple creations of 3DPT, such as Hands-Free Door Openers and Press Knobs, could prevent the spread of infectious diseases such as COVID-19. Realizing the shortage of medical equipment and opportunities of 3DPT, FDA has released FAQs on the utilization of 3D printed PPE to counter COVID-19. Companies have massively utilized 3DPT to produce and supply equipment to the hospitals. However, FDA has cited technical challenges that need to be addressed for 3DPT designed PPE to be effective. For example, 3D-printed PPE may provide a physical barrier, but 3D-printed PPE are unlikely to provide the same fluid barrier and air filtration protection as FDA-cleared surgical masks and N95 respirators [11]. Novel 3D devices have been developed by the companies [12 - 14] to mitigate COVID19.

\section{Big Data Analytics}

The BDA systems integrated with AI and TT can be used to perceive swift insights and undertake proactive decisions to encounter COVID-19 by extracting and analyzing data from

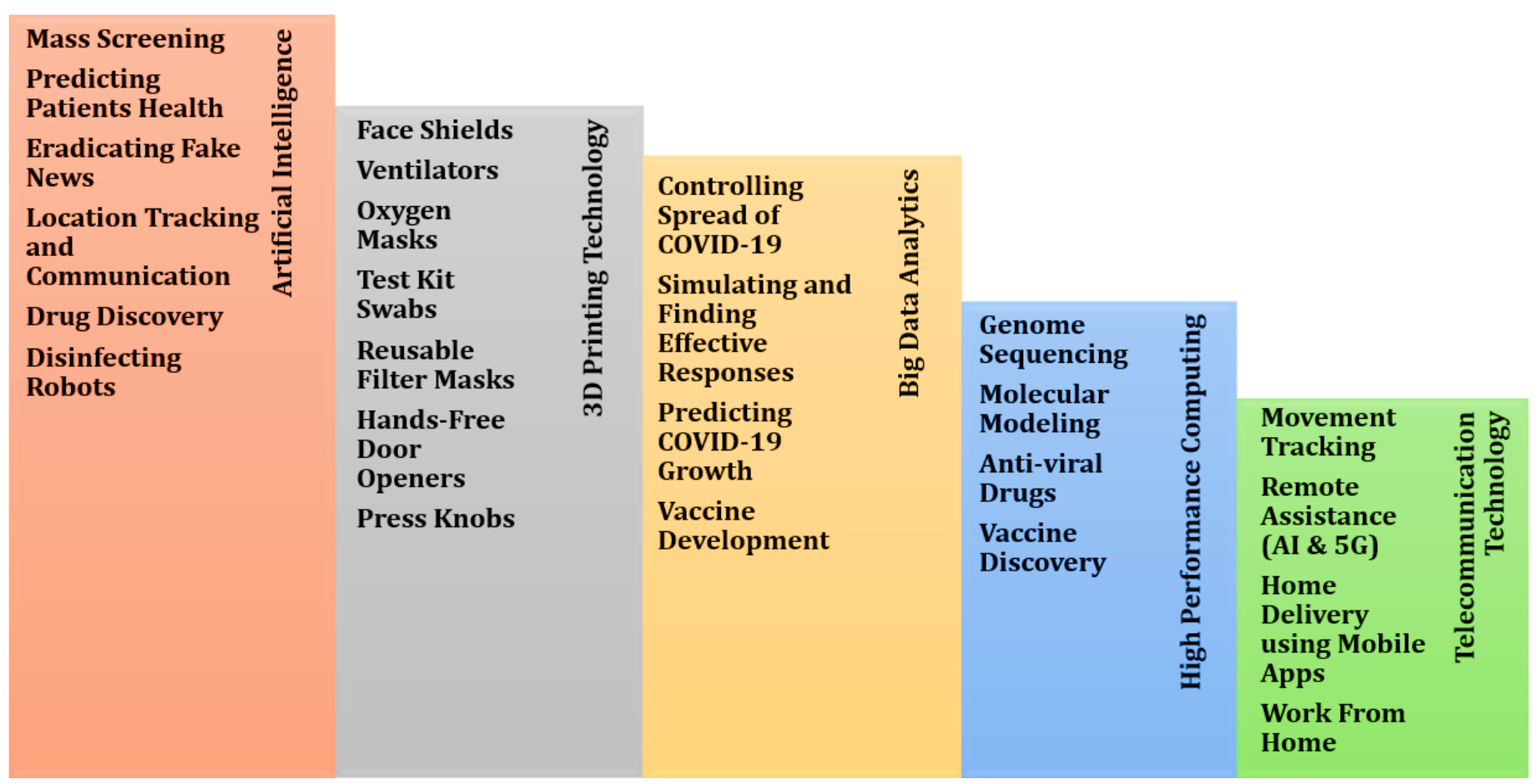

Figure 1: Key Applications of State-of-the-Art Technologies to Mitigate and Eliminate COVID-19 
sources such as hospitals, clinics, insurance, immigration and national databases, travel history, and location-tracking applications. BDA systems can categorize individuals and communities in various categories of risk. The high-risk patients, such as those with medical records of COVID-19 symptoms and recent travel history to an infectious country, can be tracked down to be quarantined immediately and undergo health screening. Similarly, the authorities can urge the low and medium risk patients such as those who have recently visited COVID-19 high-risk countries or been in potential contact with COVID-19 positive patient but are asymptomatic, to practice self-isolation. These kinds of measures taken due to insights provided by BDA systems can reduce the burden on healthcare practitioners and control the spread of disease.

Using BDA, authorities can create prescription models, simulate and analyze their impacts before implementation in real-life scenarios. Moreover, real-time trackers such as the COVID-19 map tracker by Johns Hopkins University, are analyzing huge datasets and updating the number of deaths and cases. The wealth of information generated by the trackers could be used to predict the COVID-19 curve, which can support authorities in undertaking future decisions regarding public safety and economic stability. BDA tools are also used by scientists around the world to create an anti-viral drug and vaccine against SARS-CoV-2. Some significant contributions and uses of BDA to mitigate COVID-19 are reported in [15 16].

\section{High Performance Computing}

Resolving the threats of COVID-19 requires research contributions in the domains such as bioinformatics, epidemiology, and molecular modeling, which require platforms with the massive computational capability to address complex scientific problems and process big datasets in shorter time frames. To expedite the developments, White House Office of Science and Technology Policy, the U.S. Department of Energy and IBM formed the COVID-19 HPC Consortium consisting of the federal government, academia and industry leaders who are volunteering free compute time and resources on their world-class machines [17]. Some of the consortium partners include IBM, Amazon Web Services, AMD, Google Cloud, Hewlett Packard Enterprise, Microsoft, NVIDIA, Massachusetts Institute of Technology, Rensselaer Polytechnic Institute, University of Illinois, University of Texas at Austin, Argonne National Laboratory, Lawrence Livermore National Laboratory, San Diego Supercomputer Center and NASA. Researchers can submit their proposals to the consortium to access small clusters and some of the largest supercomputers in the world. HPC can be utilized in genome sequencing, understanding the accurate biological structure of the virus and modeling various treatments. AI-driven HPC platforms can be used to discover appropriate anti-viral drugs and vaccines for COVID-19. The reports [18 - 19] have discussed the use of HPC infrastructures with supercomputers to tackle COVID-19.

\section{E. Telecommunication Technology}

Many countries have imposed lockdown to flatten the curve and contain COVID-19. Although the lockdown has severe impacts on the economy and business, it seems to be an effective process to reduce the casualties caused by the disease. TT can track individuals and assist authorities to ensure compliance with strict movement orders. With location tracking applications, AI and BDA, it is possible to alert people from visiting COVID-19 hotspots. Healthcare providers can use mobile technology to assess patients with mild symptoms remotely and provide them with guidelines during the period of self-isolation to reduce the burden on hospitals.

With the fusion of AI and 5G technology, medical robots can check patients' temperature, diagnose and provide them with recommendations with minimal intervention of healthcare professionals. TT has been the backbone of business continuity during this pandemic. TT has also made it easier for people to use numerous smartphone applications to order essential items while maintaining social distance and complying with movement control orders to stay indoors. During this pandemic, many telecommunication companies have decided to provide free services to their consumers [20 - 21]. Enterprises are complying with Work from Home (WFH) model and conducting their business, interacting with their employees as well as customers using services such as Microsoft Teams, Zoom and Skype.

\section{CONCLUSION AND FUTURE WORK}

There is a rapid demand for scientific and technical research contributions to overcome the threats of COVID-19. Due to the impact and significance of the solutions, government authorities and leading private firms have form alliances to fund and promote COVID-19 research. The technologies such as AI, 3DPT, HPC, TT and BDA have the potential to predict, mitigate and eliminate infectious diseases. The current goal of the researchers is to control the expansion curve of the COVID-19 and to eliminate it by exploring the precise vaccine. Besides threatening the global economy, this pandemic has killed many innocent lives and caused anxiety among the people, which may lead to severe health fatalities that may last even after the eradication of COVID-19.

We observed that there is shortage of research contributions in the areas of HPC to encounter COVID-19. However, we predict that the next wave of research will focus mainly on finding solutions for COVID-19 vaccination and treatment formulated from the fusion of HPC, AI and BDA. Future research also needs to focus on producing scientific solutions to monitor and ensure the well-being of the people who are living under strict movement control restrictions with social distancing and self-isolation. There is also a need for research in the areas of security and privacy issues related to technologies used for COVID-19 developments. Furthermore, technology should be utilized to assist and motivate frontline healthcare practitioners and officials in the fight against COVID-19. 


\section{REFERENCES}

[1] WHO, 2020, "Pneumonia of unknown cause - China", Retrieved from https://www.who.int/csr/don/05-january-2020-pneumonia-of-unkowncause-china/en/, Accessed on 6 April 2020.

[2] WHO, 2020, "WHO Director-General's statement on IHR Emergency Committee on Novel Coronavirus (2019-nCoV)", Retrieved from https://www.who.int/dg/speeches/detail/who-director-general-sstatement-on-ihr-emergency-committee-on-novel-coronavirus-(2019ncov), Accessed on 6 April 2020.

[3] WHO, 2020, "Novel coronavirus disease named COVID-19", Retrieved from https://www.who.int/emergencies/diseases/novel-coronavirus2019/events-as-they-happen, Accessed on 7 April 2020.

[4] WHO, 2020, "Naming the coronavirus disease (COVID-19) and the virus that causes it", Retrieved from

https://www.who.int/emergencies/diseases/novel-coronavirus2019/technical-guidance/naming-the-coronavirus-disease-(covid-2019)and-the-virus-that-causes-it, Accessed on 7 April 2020.

[5] WHO, 2020, "WHO announces COVID-19 outbreak a pandemic", Retrieved from http://www.euro.who.int/en/health-topics/healthemergencies/coronavirus-covid-19/news/news/2020/3/who-announcescovid-19-outbreak-a-pandemic, Accessed on 8 April 2020.

[6] JUH, 2020, "Coronavirus COVID-19 Global Cases by the Center for Systems Science and Engineering (CSSE) at Johns Hopkins University (JHU)", Retrieved from https://coronavirus.jhu.edu/map.html, Accessed on 11 April 2020.

[7] WHO, 2020, "Digital technology for COVID-19 response", Retrieved from https://www.who.int/news-room/detail/03-04-2020-digitaltechnology-for-covid-19-response, Accessed on 7 April 2020.

[8] Bo Ram Beck, Bonggun Shin, Yoonjung Choi, Sungsoo Park, Keunsoo Kang, 2020, "Predicting commercially available antiviral drugs that may act on the novel coronavirus (2019-nCoV), Wuhan, China through a drugtarget interaction deep learning model", bioRxiv 2020.01.31.929547; doi: https://doi.org/10.1101/2020.01.31.929547.

[9] Linda Wang, Alexander Wong, 2020, "COVID-Net: A Tailored Deep Convolutional Neural Network Design for Detection of COVID-19 Cases from Chest Radiography Images", arXiv:2003.09871.

[10] Delft Imaging, 2020, "Triage for COVID-19 Using Artificial Intelligence on Chest X-rays", Retrieved from https://www.delft.care/cad4covid/, Accessed on 10 April 2020.
[11] FDA, 2020, "FAQs on 3D Printing of Medical Devices, Accessories, Components, and Parts During the COVID-19 Pandemic", Retrieved from https://www.fda.gov/medical-devices/3d-printing-medicaldevices/faqs-3d-printing-medical-devices-accessories-components-andparts-during-covid-19-pandemic, Accessed on 11 April 2020.

[12] Materialise , 2020, "Materialise Acts: Our Response to COVID-19" Retrieved from https://www.materialise.com/en/3d-printing-response-tocovid-19, Accessed on 11 April 2020.

[13] PrinterPrezz, 2020, "Covid-19 Update" Retrieved from https://www.printerprezz.com/, Accessed on 11 April 2020.

[14] Stratasys, 2020, "Stratasys Helps: Responding to the COVID-19 Crisis" Retrieved from https://www.stratasys.com/covid-19, Accessed on 11 April 2020.

[15] Wang CJ, Ng CY, Brook RH, 2020. "Response to COVID-19 in Taiwan: Big Data Analytics, New Technology, and Proactive Testing", JAMA. Published online March 03, 2020. doi:10.1001/jama.2020.3151.

[16] Balilla, Jeffhraim, 2020. "Assessment of COVID-19 Mass Testing: The Case of South Korea (March 18, 2020)", Available at SSRN: https://ssrn.com/abstract=3556346 or http://dx.doi.org/10.2139/ssrn.3556346.

[17] COVID-19 HPC Consortium, 2020, "The COVID-19 High Performance Computing Consortium", Retrieved from https://covid19-hpcconsortium.org/, Accessed on 10 April 2020.

[18] Smith, Micholas, Smith, Jeremy C, 2020, "Repurposing Therapeutics for COVID-19: Supercomputer-Based Docking to the SARS-CoV-2 Viral Spike Protein and Viral Spike Protein-Human ACE2 Interface" ChemRxiv. Preprint. https://doi.org/10.26434/chemrxiv.11871402.v3.

[19] UCL, 2020, "Using the world's most powerful supercomputers to tackle COVID-19", Retrieved from https://www.ucl.ac.uk/news/2020/apr/using-worlds-most-powerfulsupercomputers-tackle-covid-19, Accessed on 11 April 2020.

[20] CRN, 2020, "Here's How Telecom Companies Are Helping Customers During Coronavirus", Retrieved from https://www.crn.com/slideshows/networking/here-s-how-telecom-companies-are-helpingcustomers-during-coronavirus/1, Accessed on 11 April 2020.

[21] The Star, 2020, "Covid-19: Thailand to give free mobile data for those homebound by coronavirus", Retrieved from https://www.thestar.com.my/tech/tech-news/2020/03/31/covid-19thailand-to-give-free-mobile-data-for-those-homebound-by-coronavirus, Accessed on 11 April 2020. 